\title{
Bandwidth Efficient Three-User Cooperative Diversity Scheme Based on Relaying Superposition Symbols
}

\author{
Mingwei CAO, Guangguo BI, Xiufeng JIN \\ National Mobile Communications Research Laboratory, Southeast University, Nanjing, China \\ E-mail: mwcao@163.com, \{bigg,xiufeng.jin\}@seu.edu.cn \\ Received February 3, 2009; revised February 17, 2009; accepted February 19, 2009
}

\begin{abstract}
Recently, Cooperative diversity in wireless communication systems has gained much attention. A simple two-user cooperative diversity scheme called decode-and-forward cooperation scheme has been presented by Laneman (2004). Each user has one partner to decode its information and retransmit it by employing repetition coding. This scheme can offer diversity order of two. But the bandwidth efficiency is low. In this paper, we propose a bandwidth efficient three-user cooperative diversity scheme based on relaying superposition symbols. Each user has two partners and each partner relays superposition symbols of the other two users instead of repetition. Thus, the bandwidth efficiency is improved compared to the baseline decodeand-forward cooperative diversity scheme presented by Laneman. Moreover, the proposed scheme can also offer diversity order of two. Then, in order to improve the system performance, a new constellation labeling for the superposition 8PSK modulation is designed. It is a simple way to exploit the symbol mapping diversity and a gain of about $2 \mathrm{~dB}$ can be obtained. Furthermore, the performance improvement comes at no additional power or bandwidth expense.
\end{abstract}

Keywords: Cooperative Diversity, Bandwidth Efficiency, Superposition Symbols, Symbol Mapping Diversity

\section{Introduction}

Multiple-antenna technique has been studied extensively, and it is an efficient technique to exploit spatial diversity and offer capacity gain compared to single-antenna systems [1,2]. Transmit diversity [3] has been proposed to improve the performance for systems with multiple transmit antennas.

However, in wireless communication systems, users may not be able to support multiple antennas because of the limitation of the size or cost. In this scenario, cooperative diversity, which has been proposed to achieve the transmit diversity, has gained much attention when each user only has a single antenna [4,5]. In cooperative communication systems [6,7], each user transmits its information to a destination and receives its partners' information and then serves as a relay for its partners. Hence, the destination can receive each user's information from several independent paths to efficiently resist the slowly fading effect. In baseline decode-and-forward cooperation scheme [7], two users transmit their information on orthogonal channels. Each user has one partner and decodes the partner's information and re-encodes and retransmits it through its own channel, thus, the diversity order is two. However, the bandwidth efficiency is decreased by $1 / 2$ compared to a non-cooperative diversity scheme. In order to reduce the bandwidth loss, some cooperation schemes are proposed in [8-12]. In [8], distributed space-time codes are used for cooperating transmission. Multi-source cooperation coding approach is introduced in [9-12]. In those cooperation schemes, data of multiple users are jointly encoded by relays. The bandwidth efficiency of these schemes presented in [8-12] is improved greatly, but the decoding complexity is too high.

In this paper, we propose a cooperative diversity scheme based on superposition modulation, which offers the same diversity order as the baseline decode-andforward cooperation scheme does. The bandwidth efficiency of the proposed scheme is only decreased by $1 / 3$ compared to a non-cooperative diversity scheme and the decoding complexity is much lower than that of these schemes presented in [8-12]. Moreover, a new constellation labeling for the superposition modulation is designed to improve the bit error rate (BER) performance when the system employs 8PSK modulation. Using the 
new constellation labeling, the system performance can be improved without any additional power or bandwidth expense.

Also, a cooperation scheme of two-node system based on superposition BPSK modulation is proposed in [13]. In this system, each node transmits a superposition of its own data with the data received from its partner in the previous slot. The performance of this scheme presented in [13] is further analyzed in [14,15]. In [16], the generalization of the scheme proposed in [13] to a multiple-user scenario is considered. In [17], a novel cooperation scheme based on the algebraic superposition of channel codes over a finite field is proposed. In [18], user and relay use the in-phase and the quadrature components of a QPSK signal, respectively for cooperating transmission. These schemes proposed in [13-18] are different from our scheme in which each partner retransmits the superposition symbols received from different users and each user's own symbols are not superposed with its partners' symbols.

The rest of this paper is organized as follows. In Section 2, system model is introduced, and a bandwidth efficient cooperative diversity scheme is proposed. The diversity order is discussed in Section 3 and a new symbol mapping is designed in Section 4. The simulation results are described in Section 5. Finally, conclusions are summarized in Section 6.

\section{System Model}

In this scheme, three users (A, B, and C) transmit their information to a destination (D). Assume that all channels between users and the destination (uplink channels) are Rayleigh flat slowly fading channels, which remain constant over at least six slots. We consider multiple access channels as time-division multiple access (TD MA). Thus, these users transmit in their own slots, respectively. If symbols $a_{i}, b_{i}$ and $c_{i}$, which take values from an $M$-ary alphabet $\left\{s_{0}, s_{1}, \ldots, s_{M-1}\right\}$, are transmitted from these users through three orthogonal channels, respectively, then the received baseband signals $r_{l i}, r_{2 i}$ and $r_{3 i}$ in the destination can be written as

$$
\begin{aligned}
& r_{1 i}=h_{A D} a_{i}+n_{1} \\
& r_{2 i}=h_{B D} b_{i}+n_{2} \\
& r_{3 i}=h_{C D} c_{i}+n_{3} .
\end{aligned}
$$

where $h_{A D}, h_{B D}$, and $h_{C D}$ are channel fading coefficients between users A, B, C, and the destination D, respectively, and $n_{1}, n_{2}$, and $n_{3}$ are additive noises. They are all modeled as independent complex zero-mean Gaussian random variables, and

$$
\varepsilon\left\{\left|h_{A D}\right|^{2}\right\}=\varepsilon\left\{\left|h_{B D}\right|^{2}\right\}=\varepsilon\left\{\left|h_{C D}\right|^{2}\right\}=1
$$

and

$$
\varepsilon\left\{\left|n_{1}\right|^{2}\right\}=\varepsilon\left\{\left|n_{2}\right|^{2}\right\}=\varepsilon\left\{\left|n_{3}\right|^{2}\right\}=N_{0}
$$

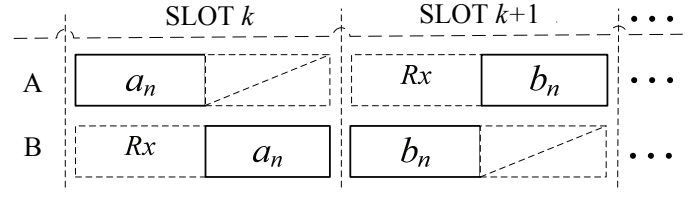

(a) System model of the baseline two-user cooperation scheme.

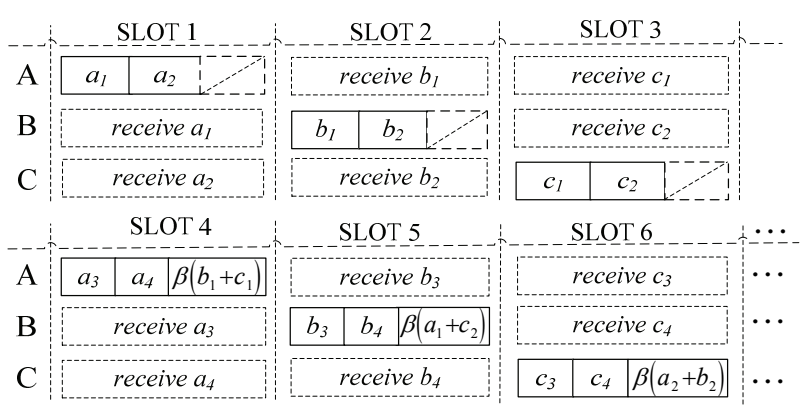

(b) System model of the proposed cooperation scheme.

Figure 1. System models of the baseline and the proposed cooperation schemes.

Note that $\varepsilon$ denotes the expectation operator.

Simultaneously, each user receives the other users' information through the channels between users (inter-user channels) and relays its partners' information only if it decodes correctly, which can be determined by the cyclic redundancy check (CRC). The destination is assumed to be informed of whether relays decode correctly or not. Generally speaking, the user is close to its partners and they may have line-of-sight connections. Thus, the quality of inter-user channels is better than that of uplink channels. In this paper, we take inter-user channels to be additive white Gaussian noise (AWGN) when line-of-sight connections exist.

\subsection{Baseline Scheme}

The decode-and-forward cooperation scheme presented in [7] is regarded as a baseline scheme. The system model of two-user cooperation scheme is shown in Figure 1 (a). Each slot is divided into two segments with the same length of time. Each user transmits its own symbols in the first segment and remains inactive in the second segment. The partner receives these symbols in the first segment, and then retransmits these symbols by employing repetition coding in the second segment if it decodes correctly, otherwise, it remains inactive.

\subsection{Proposed Scheme}

The system model of the proposed cooperation scheme is shown in Figure 1(b). Each slot is divided into three segments with the same length of time. In the first slot, user A transmits its own symbols $a_{1}$ and $a_{2}$ in the first two segments, respectively, and remains inactive in the third segment. User B only detects $a_{1}$ in the first segment and user $\mathrm{C}$ only detects $a_{2}$ in the second segment. Coopera- 
tion processes are similar in the second and third slots (see Figure 1(b)).

In the fourth slot, user A transmits its own symbols $a_{3}$ and $a_{4}$ in the first two segments, respectively, and the superposition symbol $\beta\left(b_{1}+c_{1}\right)$ in the third segment if both of $b_{1}$ and $c_{1}$ are decoded correctly, where $\beta$ is a power-normalizing scalar. In this paper, we set $\beta=\sqrt{2} / 2$ to satisfy the transmit-power constraint. If user A can only decode the symbol of user B (or user C) correctly, then user A only retransmits $b_{1}$ (or $c_{1}$ ) instead of $\beta\left(b_{1}+c_{1}\right)$ in the third segment. If neither of the symbols of user B and user $\mathrm{C}$ can be decoded correctly, then user A remains inactive in the third segment. User B detects $a_{3}$ in the first segment and user C detects $a_{4}$ in the second segment. Also, cooperation processes are similar in the fifth and sixth slots (see Figure 1(b)).

In the following slots, similar cooperation processes to those executed in the fourth to sixth slots are executed. In the last three slots, each user remains inactive in the first two segments and only retransmits the superposition symbol in the third segment.

Each user transmits its own symbols in two segments and serves as a relay for other users in only one segment. We assume that the total number of slots is large so that the loss of overhead in the first three slots can be neglected. Therefore, the bandwidth efficiency is only decreased by $1 / 3$ compared to a non-cooperative diversity scheme.

In this scheme, symbols received from two different partners are superposed, and then the superposition symbols are retransmitted from the user. This can provide diversity order of two. However, if symbols received from each partner are superposed, respectively, and then retransmitted from the user, the diversity order of two will not be achieved.

\section{Diversity Order}

Assume that perfect channel state information is available in the receiver and ideal cooperation is considered, which means each user can always decode other users' information correctly. The maximum-likelihood (ML) decoder in the destination works with pairs of transmitted symbols instead of single symbols. Thus, the decoding complexity is higher, but it is still much lower than that of the schemes presented in [8-12].

Assume that $M$-PSK ( $M \geq 4)$ modulation is employed and Gray code is used to map bits to symbols. Without loss of generality, we consider the pair of symbols $a_{i}$ and $b_{i}$, where $a_{i}, b_{i} \in\left\{s_{0}, s_{1}, \ldots, s_{M-1}\right\}$. Assume that $a_{i}, b_{i}$ and $\frac{\sqrt{2}}{2}\left(a_{i}+b_{i}\right)$ are transmitted from user A, B and C, respectively, the corresponding received baseband signals are $r_{1 i}, r_{2 i}$ and $r_{3 i}$. The receiver computes the decision metric

$$
\left|r_{1 i}-h_{A D} a_{i}\right|^{2}+\left|r_{2 i}-h_{B D} b_{i}\right|^{2}+\left|r_{3 i}-\frac{\sqrt{2} h_{C D}\left(a_{i}+b_{i}\right)}{2}\right|^{2}
$$

over all possible pairs of symbols $\left\{a_{i}, b_{i}\right\}$, and a decision is made in favor of the pair of symbols that minimizes the metric.

There will be three cases for each decision.

case 0: The decision is correct.

case 1: The decision results in one symbol error.

case 2: The decision results in two symbol errors.

The probability of case 1 for each decision is

$\operatorname{Pr}($ case 1$)$

$=\operatorname{Pr}\left(a_{i}\right.$ is decoded correctly, $b_{i}$ is decoded incorrectly $)$.

$+\operatorname{Pr}\left(a_{i}\right.$ is decoded incorrectly, $b_{i}$ is decoded correctly $)$

Equation (7) can be approximated as

$$
\begin{aligned}
\operatorname{Pr}(\text { case } 1) \approx 2 & \varepsilon\left\{Q\left(\sqrt{\frac{\left(\left|h_{A D}\right|^{2}+\left|h_{C D}\right|^{2} / 2\right) d_{\min }^{2}}{2 N_{0}}}\right)\right. \\
+ & \left.Q\left(\sqrt{\frac{\left(\left|h_{B D}\right|^{2}+\left|h_{C D}\right|^{2} / 2\right) d_{\min }^{2}}{2 N_{0}}}\right)\right\}
\end{aligned}
$$

where $Q(\cdot)$ is the Gaussian Q-function and $d_{\min }$ is the minimum Euclidean distance between pairs of signal points in the constellation. Let

$$
\gamma=d_{\min }^{2} /\left(8 N_{0}\right)
$$

then (8) can be simplified as [19]

$$
\operatorname{Pr}(\text { case } 1) \approx 2+2 \sqrt{\frac{\gamma}{1+\gamma}}-4 \sqrt{\frac{2 \gamma}{1+2 \gamma}} .
$$

when $\gamma>>1$, the probability is

$$
\operatorname{Pr}(\text { case } 1) \approx \frac{3}{8} \gamma^{-2}
$$

If the receiver decides erroneously in favor of symbols $a_{i}^{\prime} \quad\left(a_{i} \neq a_{i}^{\prime}\right)$ and $b_{i}^{\prime}\left(b_{i} \neq b_{i}^{\prime}\right)$, the value $a_{i}^{\prime}+b_{i}^{\prime}$ may be probably equal to the value $a_{i}+b_{i}$. Thus, the probability of case 2 for each decision can be upper-bounded as

$$
\begin{aligned}
\operatorname{Pr}(\text { case } 2) & <2 \varepsilon\left\{Q\left(\sqrt{\frac{\left(\left|h_{A D}\right|^{2}+\left|h_{B D}\right|^{2}\right) d_{\text {min }}^{2}}{2 N_{0}}}\right)\right\} \\
& =\frac{1}{2}\left(2+\sqrt{\frac{2 \gamma}{1+2 \gamma}}\right)\left(1-\sqrt{\frac{2 \gamma}{1+2 \gamma}}\right)^{2}
\end{aligned}
$$


when $\gamma>>1 / 2$, we obtain

$$
\operatorname{Pr}(\text { case } 2)<\frac{3}{32} \gamma^{-2}
$$

From (11) and (13), we can conclude that each erroneous decision most results in one symbol error and the average BER for each user is approximated as

$$
\begin{aligned}
p_{b} & \approx \frac{1}{\log _{2} M} \times \frac{1}{2} \operatorname{Pr}(\text { case } 1) \\
& \approx \frac{1}{\log _{2} M} \times \frac{3}{16} \gamma^{-2}
\end{aligned}
$$

which shows that the diversity order of two is achieved.

In baseline decode-and-forward cooperation scheme, the average BER for each user is approximated as

$$
p_{b}^{\prime} \approx \frac{1}{\log _{2} M} \times \frac{3}{32} \gamma^{-2} \text {. }
$$

\section{Design of Symbol Mapping}

We observed from (14) and (15) that there is signalto-noise ratio (SNR) loss for the proposed scheme compared to the baseline scheme. That is mainly because the power of the superposition symbol is equal to that of a single symbol transmitted in the first two segments in the proposed scheme. The total average energy for each symbol in the proposed scheme is 3/4 times as large as that in the baseline scheme. However, we can mitigate the SNR loss by exploiting symbol mapping diversity.

In most communication systems, multi-level modulation techniques such as $M$-PAM, $M$-PSK and $M$-QAM are employed to improve the bandwidth efficiency and the bit-to-symbol mapping for those modulation constellations is Gray code bit mapping. However, some enhanced Automatic Repeater request (ARQ) schemes [20-23] to extract additional diversity called symbol mapping diversity between retransmissions have been proposed. In those schemes, for exploiting the symbol mapping diversity, the bit-to-symbol mapping for the second transmission (first retransmission) is not Gray code bit mapping. For example, in the scheme proposed in [21], Gray code bit mapping is employed in the first transmission and another different symbol mapping is employed for maximizing the minimum combined squared Euclidean distance (CESD) [21] in the second

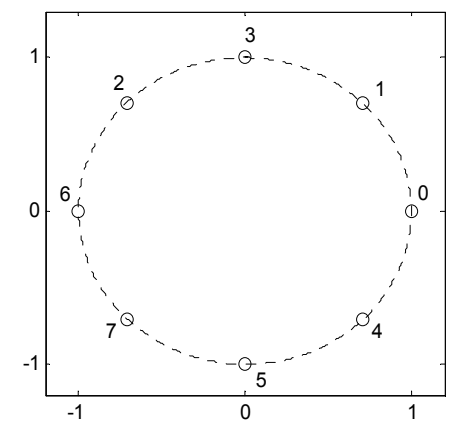

Figure 2. Signal constellation of 8PSK modulation. transmission. The method proposed in [21] is further discussed in [22] and a symbol mapping diversity scheme in Multiple-Input Multiple-Output (MIMO) systems is presented in [23]. The symbol mapping diversity can provide great BER gains in AWGN and Rayleigh flat fading channels. In this paper, we design a new symbol mapping for superposition 8PSK modulation.

The superposition symbols lead to a superposition modulation and we use Gray code bit mapping for 8PSK modulation and another symbol mapping for superposition modulation. The constellation of 8PSK modulation is showed in Figure 2. Each point represents an 8PSK symbol, which is labeled by 3 bits represented as an octal number. Since each erroneous decision most results in one symbol error and erroneous selection of an adjacent symbol, these distances between pairs of signal points, such as

$$
\left\{\begin{array}{l}
\left\{\left(s_{i} s_{j} \leftrightarrow s_{i} s_{k}\right)\right\} \\
\left\{\left(s_{j} s_{i} \leftrightarrow s_{k} s_{i}\right)\right\}
\end{array}, 0 \leq i, j, k \leq 7 \text { and } j \neq k .\right.
$$

which differ in only a symbol and two different symbols $s_{j}$ and $s_{k}$ are adjacent in 8PSK constellation, should be increased as large as possible in the superposition constellation. Based on this principle, the constellation of the superposition 8PSK modulation is designed and shown in Figure 3. Each point represents the superposition of two 8PSK symbols, which is labeled by 6 bits represented as two octal numbers.

\section{Simulation Results}

In the simulation, we assume that uplink channels are Rayleigh flat slowly fading channels. When we suppose that there exist line-of-sight connections, inter-user channels are assumed to be AWGN channels. Otherwise, inter-user channels are assumed to be Rayleigh flat slowly fading channels. In baseline decode-and-forward cooperation scheme, each user employs repetition coding to relay its partner's information. Set the symbol energy as $E s$ and assume that all inter-user channels have equal average SNR and so do all uplink channels.

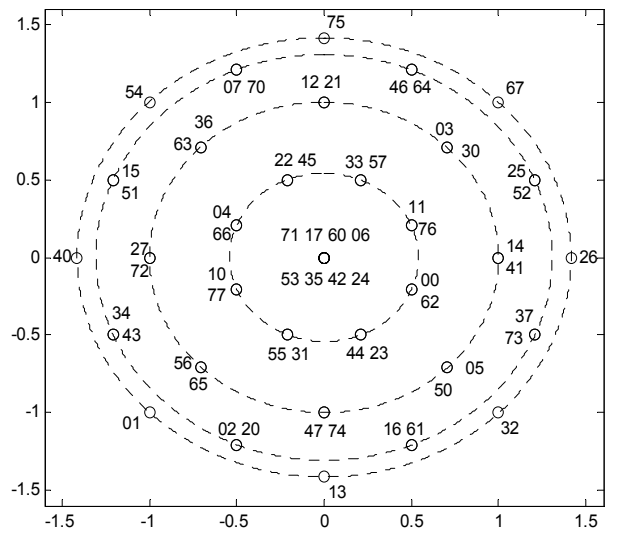

Figure 3. Signal constellation of superposition 8PSK modulation. 
Figure 4 shows the simulation results of the BER performance versus the uplink SNR per symbol $\left(E s / N_{0}\right)$ for ideal cooperation and real cooperation (all inter-user channels are assumed to be AWGN and they have equal average $\mathrm{SNR}=15 \mathrm{~dB}$ ). All cooperative diversity schemes employ QPSK modulation with Gray code bit mapping and the non-cooperative diversity scheme employs BPSK modulation. Hence, the transmission rate is $4 / 3$ bit $/ \mathrm{s} / \mathrm{Hz}$ for the proposed cooperation scheme and $1 \mathrm{bit} / \mathrm{s} / \mathrm{Hz}$ for baseline cooperation scheme and the non-cooperative diversity scheme. The analysis result of the proposed scheme is also given and it matches the simulation result very well when $E s / N_{0}$ is large and they all indicate that the diversity order of two is achieved. It is shown that the performance of real cooperation is almost the same as that of ideal cooperation when inter-user channel SNR= $15 \mathrm{~dB}$.

When line-of-sight connections do not exist, we take inter-user channels as Rayleigh flat slowly fading channels and set that inter-user channel SNR is always $10 \mathrm{~dB}$ higher than uplink channel SNR. The similar simulation is performed, and the results of the BER performance are shown in Figure 5. It is observed that the results are similar to those in Figure 4.

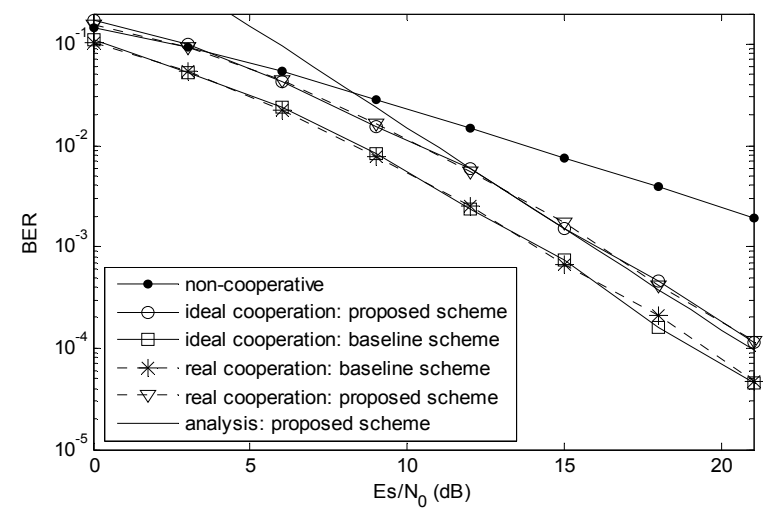

Figure 4. BER performance of ideal cooperation and real cooperation.

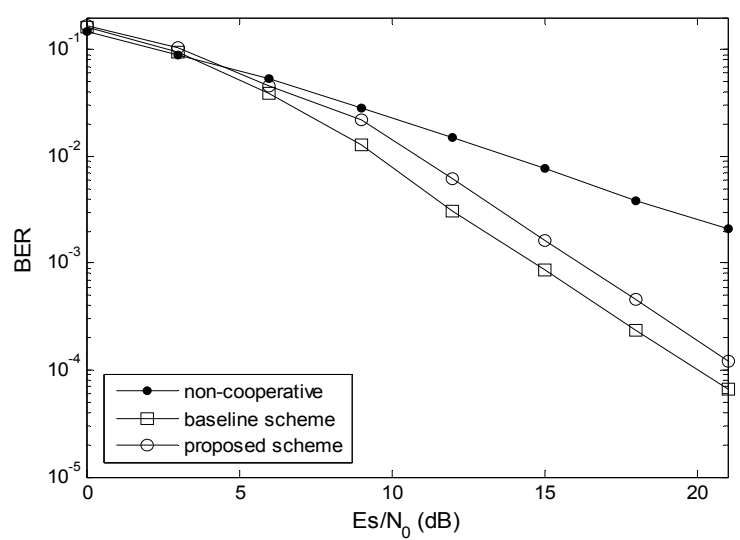

Figure 5. BER performance of cooperation schemes employing QPSK modulation and non-cooperative scheme employing BPSK modulation when inter-user channels are Rayleigh flat slowly fading channels.

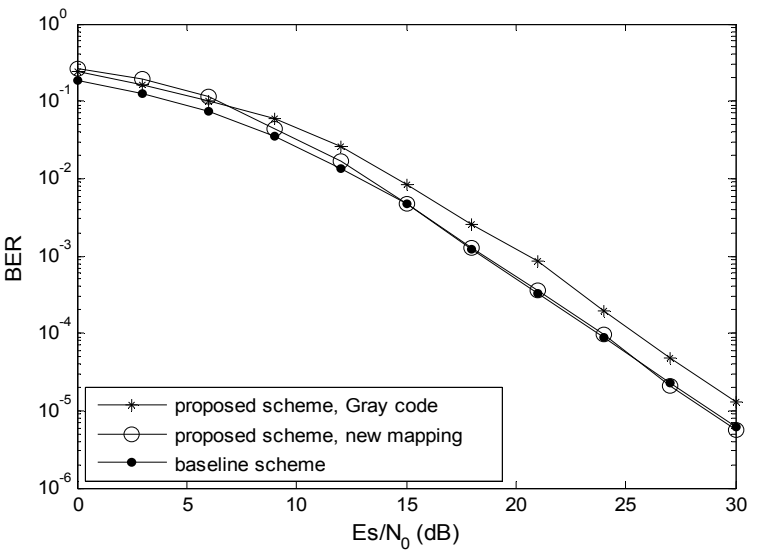

Figure 6. BER performance of cooperation schemes employing 8PSK modulation when inter-user channels are AWGN channels.

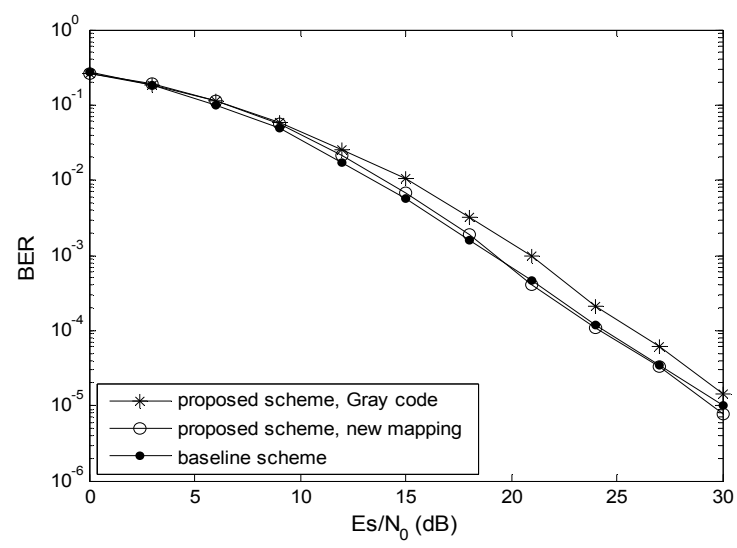

Figure 7. BER performance of cooperation schemes employing 8PSK modulation when inter-user channels are Rayleigh flat slowly fading channels.

Figure 6 shows the simulation results of the BER performance versus $E s / N_{0}$ for real cooperation (all inter-user channels are assumed to be AWGN and they have equal average $\mathrm{SNR}=20 \mathrm{~dB}$ ). All schemes employ 8PSK modulation. It is shown that there is about $2 \mathrm{~dB}$ SNR loss for the proposed scheme employing Gray code bit mapping compared to the baseline scheme. However, using the new symbol mapping, a gain of about $2 \mathrm{~dB}$ can be obtained and the performance of the proposed scheme is similar to that of the baseline scheme when $E s / N_{0}$ is large.

When inter-user channels are Rayleigh flat slowly fading channels, the simulation is also performed and the results are shown in Figure 7. Also, the inter-user channel SNR is always 10dB higher than uplink channel SNR. The proposed scheme with proposed symbol mapping has similar performance with the baseline scheme, and they both outperform the proposed scheme without symbol mapping diversity.

\section{Conclusions}

A cooperative diversity scheme using superposition modulation is proposed and a new symbol mapping is 
designed. The bandwidth efficiency of the proposed scheme is more efficient than that of the baseline decodeand-forward cooperation scheme because of superposition modulation. Moreover, it is demonstrated that the BER performance of the proposed scheme with the proposed symbol mapping is almost the same as that of the baseline cooperation scheme employing 8PSK modulation and the decoding complexity is moderate.

\section{Acknowledgement}

This work was supported by the Research Fund of National Mobile Communications Research Laboratory, Southeast University under Grant No. 2008A05, by the National Basic Research Program of China under Grant 2007CB310603, by China 863 Project under Grant No. 2007AA01Z2B1, and by NSFC Project under Grant No. 60802005 .

\section{References}

[1] G. J. Foschini and M. J. Gans, "On limits of wireless communications in a fading environment when using multiple antennas," Wireless Personal Communications, Vol. 6, No. 3, pp. 311-335, 1998.

[2] E. Telatar, "Capacity of multi-antenna Gaussian channels," European Transactions on Telecommuni- cations, Vol. 10, No. 6, pp. 585-596, 1999.

[3] S. M. Alamouti, "A simple transmit diversity technique for wireless communications," IEEE Journal on Selected Areas in Communications, Vol. 16, No. 8, pp. 1451-1458, 1998.

[4] A. Sendonaris, E. Erkip, and B. Aazhang, "User cooperation diversity Part I: System description," IEEE Transactions on Communications, Vol. 51, No. 11, pp. 1927-1938, November 2003.

[5] A. Sendonaris, E. Erkip, and B. Aazhang, "User cooperation diversity Part II: Implementation aspects and performance analysis," IEEE Transactions on Communications, Vol. 51, No. 11, pp. 1939-1948, November 2003.

[6] A. Nosratinia, T. E. Hunter, and A. Hedayat, "Cooperative communication in wireless networks," IEEE Communications Magazine, Vol. 42, No. 10, pp. 74-80, October 2004.

[7] J. N. Laneman, D. N. C. Tse, and G. W. Wornell, "Cooperative diversity in wireless networks: Efficient protocols and outage behavior," IEEE Transactions on Information Theory, Vol. 50, No. 12, pp. 3062-3080, December 2004.

[8] J. N. Laneman and G. W. Wornell, "Distributed spacetime-coded protocols for exploiting cooperative diversity in wireless networks," IEEE Transactions on Information Theory, Vol. 49, No. 10, pp. 2415-2425, October 2003.

[9] O. Shalvi, "Multiple source cooperation diversity," IEEE Communications Letters, Vol. 8, No. 12, pp. 712-714, December 2004.
[10] R. Q. Wang, W. L. Zhao, and G. B. Giannakis, "Multi-source cooperative networks with distributed convolutional coding," 2005 Conference Record of the Thirty-Ninth Asilomar Conference on Signals, Systems and Computers, pp. 1056-1060, 2005.

[11] R. Q. Wang, W. L. Zhao, and G. B. Giannakis, "Distributed trellis coded modulation for multi-source cooperative networks," Radio and Wireless Symposium, 2006 IEEE, San Diego, CA, pp. 271-274, January 2006.

[12] R. Q. Wang and G. B. Giannakis, "Complex field coding in multi-source cooperative networks for full diversity," 2006 IEEE International Conference on Communications, Vol. 10, pp. 4485-4488, June 2006.

[13] E. G. Larsson and B. R. Vojcic, "Cooperative transmit diversity based on superposition modulation," IEEE Communications Letters, Vol. 9, No. 9, pp. 778-780, September 2005.

[14] Z. G. Ding, T. Ratnarajah, and C. Cowan, "On the performance of superposition cooperative diversity in wireless networks," 2006 IEEE International Symposium on Information Theory, pp. 2714-2718, July 2006.

[15] K. Ishii, "Cooperative transmit diversity utilizing superposition modulation," Radio and Wireless Symposium, 2007 IEEE, pp. 337-340, January 2007.

[16] Z. G. Ding, T. Ratnarajah, and C. C. F. Cowan, "On the divesity-multiplexing tradeoff for wireless cooperative multiple access systems," IEEE Transactions on Signal Processing, Vol. 55, No. 9, pp. 4627-4638, September 2007.

[17] L. Xiao, T. E. Fuja, J. Kliewer, and D. J. Costello, "Cooperative diversity based on code superposition," 2006 IEEE International Symposium on Information Theory, pp. 2456-2460, July 2006.

[18] V. Mahinthan, J. W. Mark, and X. M. Shen, "A cooperative diversity scheme based on quadrature signaling," IEEE Transaction on Wireless Communications, Vol. 6, No. 1, pp. 41-45, January 2007.

[19] M. K. Simon and M. Alouini, "A unified approach to the performance analysis of digital communication over generalized fading channels," Proceedings of IEEE, Vol. 86, No. 9. pp. 1860-1877, September 1998.

[20] C. Wengerter, A. G. Edler von Elbwart, E. Seidel, G. Velev, and M. P. Schmitt, "Advanced hybrid ARQ technique empolying a signal constellation rearragement," in Proceedings of IEEE VTC 2002 Fall, Vol. 4, pp. 2002-2006, September 2002.

[21] H. Samra, Z. Ding, and P. M. Hahn, "Symbol mapping diversity design for multiple packet transmissions," IEEE Transactions on Communications, Vol. 53, No. 5, May 2005.

[22] M. Gidlund, "Performance of hybrid ARQ scheme based on bit-to-symbol mapping," 2008 IET International Conference on Wireless, Mobile and Multimedia Networks, pp. 303-306, January 2008.

[23] S. H. Moon, H. H. Park, A. Goldsmith, and M. S. Oh, "Bit rearrangement for MIMO retransmissions," Global Telecommunications Conference, 2007, GLOBECOM '07 IEEE, pp. 3509-3513, November 2007. 